\title{
Wire Materials for Scanners in the Large Hadron Collider: an Unusual Materials Selection Problem
}

\author{
Hal Bigland ${ }^{1}$, John E. Huber ${ }^{*}$, Ray Veness ${ }^{2}$, Alan C.F. Cocks ${ }^{1}$ \\ ${ }^{1}$ Department of Engineering Science, University of Oxford, Parks Rd. Oxford OX1 3PJ. \\ ${ }^{2}$ Beam Instrumentation Group, CERN, CH-1211 Geneva 23. \\ *Corresponding author. Email: john.huber@eng.ox.ac.uk
}

\begin{abstract}
The transverse distribution of particles in the hadron beam of the Large Hadron Collider (LHC) is measured using wire scanners that pass a thin wire through the hadron beam and detect scattered radiation. Increasing demands on beam instrumentation in the LHC lead us to consider the choice of material for the wire. Here, it is shown that this material choice depends on thermal, mechanical and radiation scattering properties. Simple analytical models are used to develop an Ashby material index for the design problem. It is confirmed that carbon fibres, currently used in several scanners, are high-performing materials for this application. The key compromises in the material choice are identified. Notably, thermal conductivity and emissivity are relatively unimportant, while heat capacity, tolerance of high temperature and mechanical strength dominate the material index. Potential competitor materials are identified, along with directions to pursue for performance enhancement.
\end{abstract}

\section{Introduction}

The Large Hadron Collider at CERN accelerates protons and other hadrons such as lead ions to energies up to $7 \mathrm{TeV}$ and stores them in a ring of $27 \mathrm{~km}$ circumference where they collide in the centre of dedicated particle physics experiments ${ }^{[1]}$. The LHC is the final, high-energy part of an 'injector chain' of five accelerators that take particles from rest through different energy steps, analogous to the gears in a car.

The particle beams have a transverse distribution that is quasi-Gaussian across the physical aperture whose width varies over typically one order of magnitude depending on particle energy, position in the accelerator and critically, errors during the acceleration process or transfer between accelerators. Understanding the distribution of particles in each stage of this 
acceleration is essential in the production of the narrow, high intensity beams required by the LHC experiments.

The principal instrument, used in all five of the accelerators of this chain is the 'wire scanner'. This functions by passing a fine wire across the circulating beam. The interaction between the wire material and the particles scatters some of the particles from their trajectory and produces a 'shower' of secondary, lower energy particles by elastic and inelastic collision. ${ }^{[2]}$ This flux of secondary particles is measured outside of the beam vacuum enclosure, normally with a scintillator and photo-detector. ${ }^{[3]}$ Combining the variation of the particle flux signal in time with a precision measurement of the wire position allows a direct reconstruction of the particle distribution in one plane to be made. Combining data from horizontal and vertical instruments gives the full transverse particle distribution at a given location.

There are a number of different designs of wire scanners in operation at CERN. The engineering design challenges typically increase with the energy of particles as they progress through the accelerator chain. Higher energy beams have a smaller transverse size (with a typical standard deviation of $0.5 \mathrm{~mm}$ for the LHC beams) so instruments need a better resolution. At energies above $1 \mathrm{GeV}$, further increase in energy can also make the scanned beams more destructive to the wire material.

The solution to this problem is to use fine wires with excellent high-temperature performance and scan them rapidly across the beam with a precise position measurement. The latest generation of wire scanners being developed at CERN are designed for a $20 \mathrm{~ms}^{-1}$ transition speed and a position measurement accuracy of $\sim 10 \mu \mathrm{m} .{ }^{[3]}$ Currently used wires are $30 \mu \mathrm{m}$ diameter carbon filaments. However, even with these parameters, it is not possible to scan the full beam in the LHC without rapidly destroying the wire, which fails by erosion through sublimation.

The LHC and injectors are in the process of upgrade which will increase beam intensity and decrease beam sizes across the chain. This will further reduce the scope of these key instruments. It was therefore decided to review the choice of materials for the wire, returning to first principles and using modern materials selection methods to review the full range of possible wire materials along with novel materials. This article develops simplified thermal and mechanical models of the principal loads on the wire. These are then used to create an 'Ashby plot' to show the relative merits of different candidate materials in this specific thermomechanical domain. 


\section{The materials selection problem}

Ashby ${ }^{[4,5]}$ developed a systematic method for selecting the material best suited to a defined set of engineering requirements. This typically consists of the derivation of a material index - a combination of material properties maximized in the best material for the application. An example of such an index is $\sigma_{\mathrm{f}}{ }^{k} / \rho$ for the selection of light, strong structural elements. Here, $\sigma_{\mathrm{f}}$ is the failure stress in brittle materials or elastic limit in ductile materials, $\rho$ is the mass density and $k$ is an application dependent constant equal to unity for tensile structural elements, two for linear bending elements and three for plates. The material index is derived by a formal optimization in which the objective function and constraints define the design problem to be solved.

Considerable simplification and approximation of design problems is made when deriving indices for materials selection. As material properties vary by orders-of-magnitude, some details of the design problem will have negligible effect on the choice of material. For example, in the selection of material for a lightweight spring with high mechanical energy storage, the optimal choice of material is largely independent of the geometry and size of the spring. There are intrinsically good materials for springs ${ }^{[4]}$. The analysis here is in the same spirit: approximations will be made that at first sight may seem to compromise the accuracy of the analysis, but the key physics of the design problem is preserved. Including too much detail renders the problem analytically intractable, and prevents the development of material indices. However, with suitable simplifications, analytical results can be found that correctly capture the scaling of performance measures with material properties.

The problem of selecting a material for the wire scanner can be defined by identifying an objective function and constraints. There is currently demand for increased energy and luminosity from the $\mathrm{LHC}^{[1]}$, so we define an objective of maximizing the greatest power of hadron beam that can be scanned without material failure. A material failure occurs if the wire breaks under load due to softening, melting, or sublimation. In practice, it may be desirable to limit the range of energies at which the hadron beam is scanned, so as to control the number of reliable scans before failure; this does not affect the material choice. Let the beam power be

$$
P_{\text {beam }}=\dot{n} E
$$


where $\dot{n}$ is the particle flux (particles per second) and $E$ is the typical energy per particle. The objective is to maximize $P_{\text {beam }}$ subject to certain constraints. A first constraint is

$$
T_{\mathrm{p}}<T_{\max }
$$

where $T_{\mathrm{p}}$ is the peak temperature of the wire reached during scanning and $T_{\max }$ is a materialdependent limiting temperature. For many materials the softening temperature is an appropriate choice, but melting temperature could be used as an alternative, noting the ready availability of melting temperature data. A second constraint is

$$
\sigma_{\mathrm{p}}<\sigma_{\mathrm{f}}
$$

where $\sigma_{\mathrm{p}}$ is the peak tensile stress in the wire during scanning and $\sigma_{\mathrm{f}}$ is a material dependent failure stress. The appropriate failure stress is the tensile strength in the case of brittle wire while the yield stress may be used for ductile wire. The origin of tensile stress in the wire during the scan is twofold: A static tension is applied, normally by bending the supporting forks, to hold the wire straight. Additionally, inertial load is imposed due to acceleration of the wire during the scanning process. Both aspects will be considered.

Finally, there is a third constraint imposed by metrology requirements. The motion of the wire during each scan induces vibration, leading to uncertainty in the position of the wire and hence a limitation on the precision of position measurements. The vibrations are initiated by the inertial loading of the wire as it accelerates from rest to the scanning speed (note that fluid drag forces are negligible since the operation is in high vacuum). Let the amplitude of deflection due to inertial loading be $\delta_{\mathrm{p}}$. This amplitude is constrained to be less than a limit $\delta_{\max }$ imposed by the required precision of the device, typically about $5-10 \mu \mathrm{m}^{[3,6]}$ :

$$
\delta_{\mathrm{p}}<\delta_{\max }
$$

At first sight, the objective and constraints (1-4) appear unrelated. However, they couple together via the physics of the problem as follows. Deposition of energy, proportional to the beam power (1) governs the heating of the wire, and hence relates to the peak temperature (2). This temperature can be reduced by scanning faster, since the increased speed reduces the time of exposure of the wire to the hadron beam. However, this can only be achieved at the cost of greater acceleration, since the angular range of the scanner forks is limited. Greater acceleration increases the inertial loading and thus increases the deflection of the wire: it is constrained by 
(4). Deflection of the wire can be resisted by pre-tensioning, but this is limited by the constraint on failure, (3). Simple models for each of these physical interactions are needed in order to develop a material index for the selection problem.

\subsection{Thermal model}

The wires used in the LHC are known to increase in temperature by of the order of $10^{3} \mathrm{~K}$ during scanning. ${ }^{[7]}$ Thus, in addition to considering the heat capacity of the wire and conduction along the wire as a potential source of cooling, other types of cooling including radiative cooling, thermionic emission and material evaporation or sublimation may also be significant. Sapinski ${ }^{[7]}$ developed a model of thermal energy absorbed in the wire based on these phenomena, leading to:

$$
P=\rho V c_{\mathrm{p}}(T) \frac{\partial T}{\partial t}+A_{\mathrm{rad}} \varepsilon \sigma\left(T^{4}-T_{\mathrm{amb}}^{4}\right)+P_{\mathrm{cond}}+P_{\mathrm{th}}+P_{\mathrm{cur}}+P_{\mathrm{sub}}
$$

where $P$ is the total thermal power absorbed by the wire, deposited by the hadron beam. This will later be related to the total beam power. In the first power absorption term, $\rho V c_{\mathrm{p}}(T)$ is the thermal mass of the heated volume $V$ of wire, having density $\rho$ and heat capacity $c_{\mathrm{p}}(T)$ at temperature $T$. Here $\frac{\partial T}{\partial t}$ is the rate of temperature increase. Hence this first term gives the power absorbed in heating the wire. The second term gives the radiated heat: $A_{\text {rad }}$ is the surface area of the region of wire radiating heat, $\varepsilon$ is the emissivity of the wire, $\sigma$ is the StefanBoltzmann coefficient, and $T_{\text {amb }}$ is the ambient temperature. The subsequent terms represent heat loss by conduction along the wire, $P_{\text {cond }}$, by thermionic emission, $P_{\text {th }}$, the heat generated due to a current compensating the thermionic emission, $P_{\text {cur }}$, and heat loss due to sublimation or evaporation $P_{\text {sub }}$. Each of these four terms was assessed to be small enough to neglect in generating the material selection model, though they can collectively make a significant difference to the thermal history experienced by the wire. Specifically, the time scale for thermal conduction along the wire depends on the wire's thermal diffusivity, $a$, with units of $\mathrm{m}^{2} \mathrm{~s}^{-1}$ and the distance along the wire that heat must conduct in order to escape from the heated zone, which scales with the hadron beam diameter $D$ of around $250 \mu \mathrm{m}-500 \mu \mathrm{m}$. From dimensional considerations alone, the time scale for thermal diffusion is $D^{2} / a$ times a 
dimensionless constant of order unity. Since solids have thermal diffusivity in the range $10^{-8}$ $10^{-4} \mathrm{~m}^{2} \mathrm{~s}^{-1},{ }^{[8]}$ the resulting range of time scales is from milliseconds up to seconds. Comparing this with transit times for the wire crossing the hadron beam, which are of order $10^{-4} \mathrm{~s}$ indicates that $P_{\text {cond }}$ would be significant only in the most conductive wire materials, and then only for the slowest transits. Almost always, $P_{\text {cond }}$ can be neglected for a first approximation. Thermionic emission and the related compensating current are significant during cooling from the peak temperature, but do not greatly affect the peak temperature reached, which is the focus of the analysis. Sublimation and evaporation should generally be minimised for a long wire life. Although they are significant in generating damage to the wires, the heat loss by this mechanism is small.

For simplicity, the power input was treated as a constant while the beam irradiates the wire. The specifics of the beam profile and distribution of energy deposition will be unchanged from material to material and have negligible effect on the material selection problem. It is convenient to normalize temperature in equation (5) by introducing $\bar{T}=T / T_{\mathrm{e}}$ where $T_{\mathrm{e}}$ is the equilibrium temperature at which the input power is just balanced by radiated heat loss, $T_{\mathrm{e}}=\left(\frac{P}{A_{\mathrm{rad}} \varepsilon \sigma}\right)^{1 / 4}$. Similarly, time can be normalized by introducing $\tau=t / t_{\mathrm{e}}$ where $t_{\mathrm{e}}=\rho V c_{\mathrm{p}} T_{\mathrm{e}} / P$. A normalized time $\tau$ equal to unity corresponds to a time such that steady heating with power $P$ brings the wire temperature up to the equilibrium temperature $T_{\mathrm{e}}$. Then, integrating equation (5) gives the thermal evolution of the heated part of the wire as:

$$
\tau=\frac{1}{2}\left(\tan ^{-1} \bar{T}+\tanh ^{-1} \bar{T}\right)
$$

This result is shown in figure 1a. Equation (6) indicates two distinct regimes of the thermal response that may occur: (i) an essentially linear heating regime when $\tau \ll 1$, where $\bar{T} \approx \tau$, and (ii) a steady state regime when $\tau \gg 1$ and $\bar{T} \approx 1$. If the transit of the wire across the beam is sufficiently rapid, the heating response remains within regime (i) and the peak normalized temperature varies inversely with transit speed. For sufficiently slow transits, regime (ii) is reached and the wire temperature stabilizes due to radiative losses. In this latter case, the approximation made in neglecting thermionic emission is relatively crude and equation (6) 
may be expected to overestimate the peak temperature. The maximum error between the linear approximations (i), (ii) and equation (6) is $14 \%$.
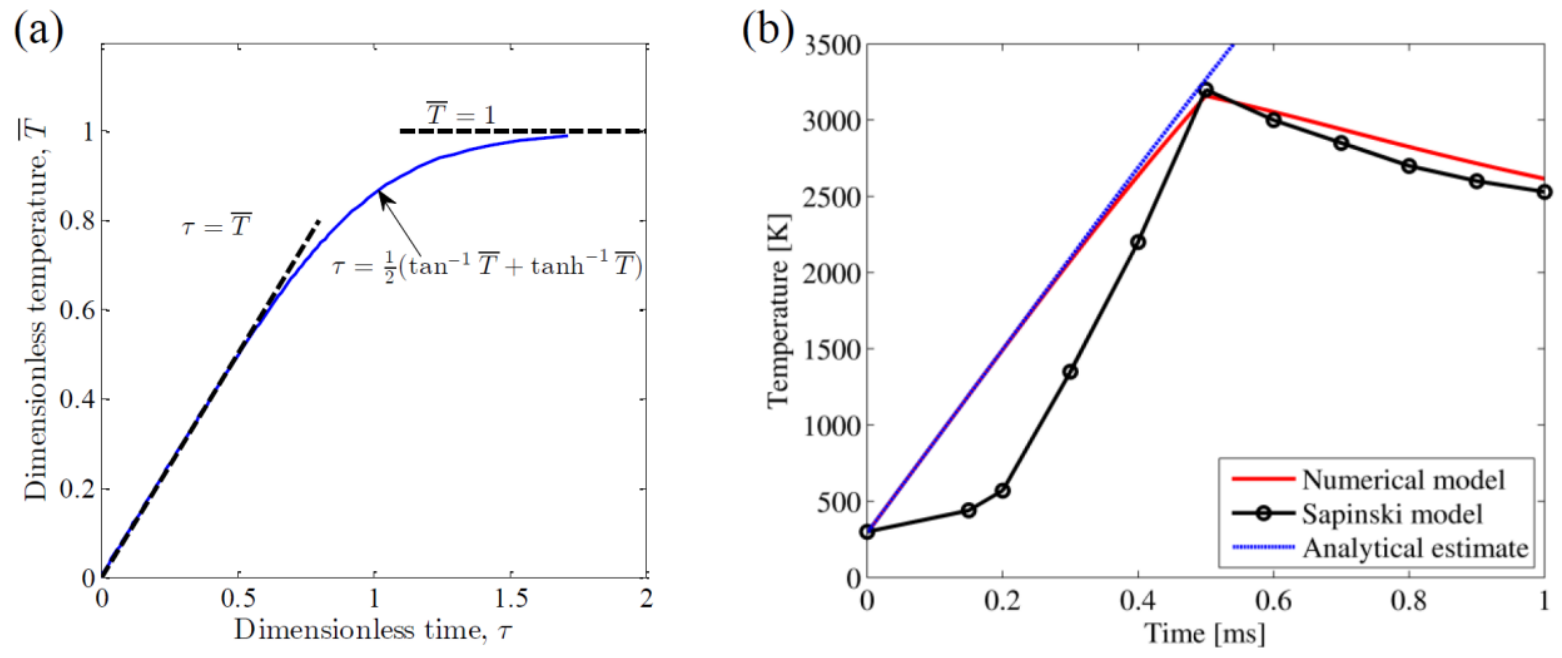

Figure 1. Models of the thermal response of the wire. (a) Analytical model including radiative cooling only. (b) Comparison of the analytical model with two numerical simulations for the case of a $34 \mu \mathrm{m}$ carbon fibre wire subject to $3.5 \mathrm{~W}$ power input for $0.5 \mathrm{~ms}$.

\begin{tabular}{ll}
\hline Density $\rho\left[\mathrm{kg} \mathrm{m}^{-3}\right]$ & 1800 \\
Specific heat capacity c $\left[\mathrm{J} \mathrm{kg}^{-1} \mathrm{~K}^{-1}\right]$ & 712 \\
Emissivity $\varepsilon[-]$ & 0.8 \\
Wire diameter $d[\mu \mathrm{m}]$ & 34 \\
Beam diameter $D[\mu \mathrm{m}]$ & 500 \\
Deposited power $P[\mathrm{~W}]$ & 3.5 \\
Wire velocity $v\left[\mathrm{~m} \mathrm{~s}^{-1}\right]$ & 1 \\
Thermal conductivity $\lambda\left[\mathrm{W} \mathrm{m}^{-1} \mathrm{~K}^{-1}\right]$ & 30 \\
\hline
\end{tabular}

Table 1. Material properties for the carbon fibre and other parameters assumed in the analytical model and numerical model.

As an illustration of the ability of the analytical estimate to capture the thermal history of the wire, figure $1 \mathrm{~b}$ shows the analytical model and the results of two other simulations. The case studied is the thermal loading of a $34 \mu \mathrm{m}$ carbon fibre traversing a $500 \mu \mathrm{m}$ hadron beam at velocity $v=1 \mathrm{~ms}^{-1}$. This speed was chosen for direct comparability with the numerical results 
of Sapinski. ${ }^{[7]}$ The average input thermal power is assumed to be $3.5 \mathrm{~W}$. Material properties and other parameters in the model are given in table 1.

A numerical model is labelled in figure $1 \mathrm{~b}$; this shows a solution to equation (5) including conduction along the wire, but excluding the thermionic emission, compensating current and sublimation terms. The governing equation then becomes

$$
\frac{P}{D}=\rho c_{\mathrm{p}} \frac{\pi d^{2}}{4} \frac{\partial T}{\partial t}+\pi d \varepsilon \sigma\left(T^{4}-T_{\mathrm{amb}}^{4}\right)+\lambda \frac{\pi d^{2}}{4} \frac{\partial^{2} T}{\partial z^{2}}
$$

where $d$ is the wire diameter, $D$ is the diameter of the hadron beam, $\lambda$ is the thermal conductivity of the wire, and $z$ is a position variable indicating position along the wire. All other variables and constants are as previously defined.

In the numerical model, the power input was assumed to be uniformly distributed over a region of the wire of length equal to the beam diameter, $D$, and constant in time for the duration of transit $D / v$. For simplicity, the heat capacity was treated as a constant, using a value representative of the range of temperatures reached. The solution was computed by discretizing the wire into elements of length $1 \mu \mathrm{m}$, and discretizing time in steps of $0.2 \mu \mathrm{s}$. The temperature shown in figure $1 \mathrm{~b}$ corresponds to the midpoint of the heated segment of wire. Also shown, labelled 'Sapinski model', is a set of points taken from a report of Sapinski, ${ }^{[7]}$ based on a model that includes all of the terms in equation (5), and uses a realistic distribution of intensity over the cross-section of the beam. Sapinski's model also accounts for variation in both the specific heat capacity of the wire, and its thermal conductivity, with temperature. The result of this more sophisticated numerical model shows a gradual start to the heating process, followed by more rapid heating as the centre of the hadron beam crosses the wire. However, as figure $1 \mathrm{~b}$ shows, the peak temperature reached during the heating process is similar in all three models. Note that, in the analytical estimate, $\tau \ll 1$ in the region shown; the temperature-time graph is thus almost a straight line. The peak temperature is the temperature reached at the end of the heating process $(t=0.5 \mathrm{~ms}$ in the case modelled; the analytical estimate is not valid beyond this point).

As there can be uncertainty in some of the material properties, we next explore the sensitivity of the predicted peak temperature to several parameters, using the numerical model. The numerical model was re-run with variations in thermal conductivity $\lambda$, heat capacity $\rho \mathrm{c}_{\mathrm{p}}$ and emissivity $\varepsilon$ by $\pm 25 \%$ based on the nominal values used to generate figure $1 \mathrm{~b}$. The percentage 
variation in the peak temperature reached is shown in figure 2. This shows that increases in $\lambda, \rho \mathrm{c}_{\mathrm{p}}$ or $\varepsilon$ reduce the peak temperature. However, the peak temperature is insensitive to errors in the conduction and radiative cooling terms: its value is dominated by the thermal mass of the wire. Thus the assumption of regime (i), with $\bar{T} \approx \tau$, neglecting cooling, is reasonable. This gives rise to a very simple estimate of the peak temperature in the wire as:

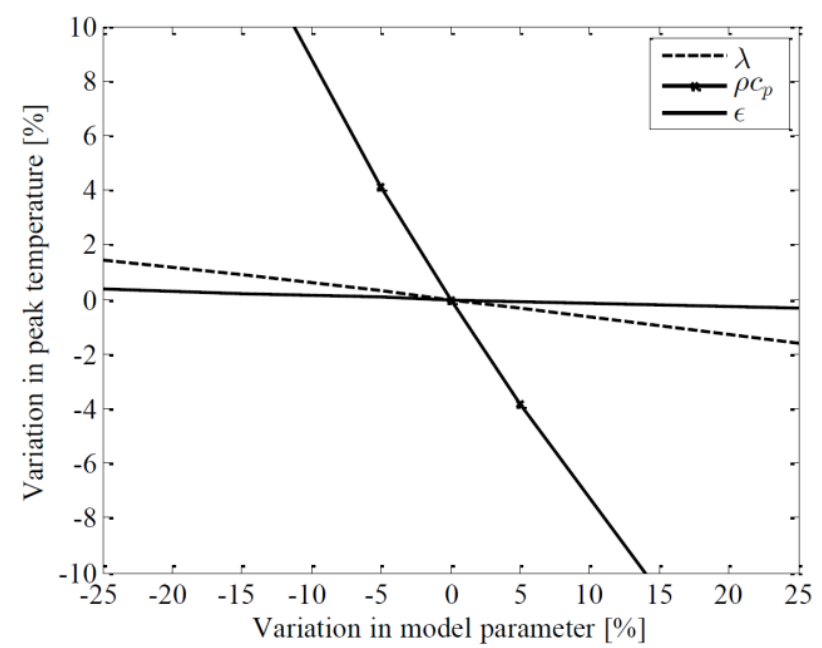

Figure 2. Variation in estimated peak temperature due to changes in thermal conductivity, heat capacity, or emissivity.

$$
T_{\mathrm{p}}=\frac{4 P}{\rho \mathrm{c}_{\mathrm{p}} \pi d^{2} v}
$$

The value of heat capacity $\rho c_{p}$ used here should be a representative average over the range of temperatures encountered.

The thermal power $P$ absorbed from the hadron beam is strongly dependent on the nuclear properties of the wire material, as well as the wire thickness and beam diameter. The physics of energy transfer from a hadron beam into matter entails several distinct processes. At high energies, nuclear interaction becomes a negligible fraction of the total thermal power input. By contrast, electronic interactions transfer momentum into the wire and provide the majority of heating. The stopping power $-\mathrm{d} E / \mathrm{d} x$, often approximated by the Bethe formula ${ }^{[9]}$, indicates the energy loss per unit path length and is dependent on the particle energy. Since interactions of the high energy particles with matter produce cascades of further events, accurate calculation 
of the total stopping power is typically achieved using simulations of the multiple interactions that can occur. ${ }^{[9]}$ Alternatively, at high particle energies, the Bethe formula has sufficient accuracy. For the wire scanner application, the restricted stopping power, which excludes energy loss due to secondary electrons, is the appropriate measure of thermal power. Since the wire thickness is much less than the typical range of protons at the LHC injection energy in solids, which is of order meters, we consider only the initial value of restricted stopping power at the typical particle energy $E$, which is expected to be in the range $100 \mathrm{GeV}-10 \mathrm{TeV}$. Restricted stopping power values were computed for this range of energies and for a variety of materials using the FLUKA Monte-Carlo simulation code ${ }^{[10]}$ and an energy cut-off of $\Delta=30 \mathrm{keV}$. The energy cut-off defines a maximum energy of secondary electrons included in the calculation, such that electrons with energy greater than $\Delta$ are ignored. In practice, the calculation of energy absorbed is insensitive to the exact value of this cut-off because highenergy secondary electrons typically have a range much greater than the wire diameter. Two key results from the FLUKA simulations are that, for this range of energies, the restricted stopping power varies approximately linearly with material density and is almost independent of the particle energy. The path length through the wire can be approximated by the wire diameter, $d$, and the length of wire exposed to the beam is determined by the beam diameter, $D$. Then the absorbed power, based on a circular beam of uniform density, is approximately

$$
P=\frac{\dot{n}}{\pi D^{2} / 4} \frac{\mathrm{d} E}{\mathrm{~d} x} D \pi d^{2} / 4=\frac{P_{\text {beam }}}{E D} \Omega \rho d^{2}
$$

where $\Omega$ is the approximately constant ratio of restricted stopping power to density, known as the "mass stopping power". Combining equations (8) and (9) gives a peak temperature of

$$
T_{\mathrm{p}}=\left(\frac{4 P_{\text {beam }} \Omega}{\pi E D}\right)\left(\frac{1}{v \mathrm{c}_{\mathrm{p}}}\right)
$$

where the physical constants and beam parameters have been separated from the scanner design variables for clarity. It is interesting that this expression appears independent of the wire diameter $d$. This can be understood as follows: consider replacing a single wire with a bundle of similar wires. To the order of approximation made, each member of the bundle of wires will experience identical heating and the peak temperature is unchanged. The analysis is valid provided $\frac{\mathrm{d} E}{\mathrm{~d} x} d \ll E$ and $D \ll v t_{\mathrm{e}}$. The former inequality gives the condition that the wire 
diameter is much smaller than the decay path length, while the latter inequality ensures that the wire transits across the beam well before thermal equilibrium is reached. For slow transits, with $D \geq v t_{\mathrm{e}}$, the peak temperature is dependent on cooling.

\subsection{Mechanical model}

The scanned wire is supported on a pair of compliant fork-arms under a tension $H \sim 0.5 \mathrm{~N}$, which holds it straight. The fork-arms then rotate about a fixed centre, moving the wire along an arc of radius $R$, typically of $100-200 \mathrm{~mm}^{[3]}$. In a typical scanner, the fork-arms turn through about $225^{\circ}$, accelerating the wire to a peak speed $v$ and maintaining that speed over a $45^{\circ}$ arc before decelerating to rest, see figure 3 . Inertial loading causes a peak deflection of the wire, $\delta_{\mathrm{p}}$, from its straight configuration. Defining the angular position, velocity and acceleration of the forks as $\theta, \omega$ and $\dot{\omega}$ respectively, the magnitude of instantaneous acceleration of the wire is

$$
a=R \sqrt{\omega^{4}+\dot{\omega}^{2}}
$$

During the process of bringing the wire up to full speed, the angular acceleration of the forks varies from zero, up to a maximum $\dot{\omega}_{\text {max }}$, and back to zero as the steady scanning speed is attained. A simple model of smooth angular acceleration variation, with peak angular velocity $\omega_{\max }$ reached after a period of acceleration $t_{\mathrm{a}}$, and maximum angular acceleration $\dot{\omega}_{\max }$, is given by

$$
\dot{\omega}=\frac{\dot{\omega}_{\max }}{2}\left(1-\cos 2 \pi \frac{t}{t_{\mathrm{a}}}\right)
$$

The peak angular velocity and peak angular acceleration do not occur at the same instant (see figure $4 \mathrm{a}$ ), but can be related, using (12), by

$$
\omega_{\max }^{2}=\Phi \dot{\omega}_{\max }
$$

where $\Phi$ is the angular range over which the acceleration occurs. This angular range is typically around $\pi / 2$, so the tangential acceleration component in (11) is smaller than the centripetal component, but not negligible. However, as illustrated in figure $4 \mathrm{~b}$, the maximum acceleration is simply $R \omega_{\max }^{2}$ provided $\Phi>\pi / 3$, which is normally the case. 
The peak deflection of the wire may then be estimated by treating it as a cable held between end supports and subjected to a uniform transverse loading $w=\left(\rho \pi d^{2} / 4\right) R \omega_{\max }^{2}$ per unit length. Gravitational loading has been neglected: it is significant for some current designs with $R \sim 0.1 \mathrm{~m}$ and $v \sim 1 \mathrm{~ms}^{-1}$, but becomes negligible for faster scans with $v>3 \mathrm{~ms}^{-1}$. Current design targets have $v \sim 10-20 \mathrm{~ms}^{-1} \cdot{ }^{[3]}$ Using uniform loading and elementary cable theory gives the maximum deflection as $w L^{2} / 8 H$, where $L$ is the length of the wire between the supporting forks. Note that the flexural rigidity of the wire has been neglected as this scales with the fourth power of the wire diameter, resulting in a vanishingly small influence for fine wires. Similarly, the fractional variation in $L$ due to inertial loading may be neglected as this scales with $\left(\delta_{\mathrm{p}} / L\right)^{2}$, which is typically of order $10^{-8}$ or less. It should also be noted that the inertial contribution to the wire tension is typically negligible by comparison with the pre-load used to keep the wire approximately straight. Then, noting that $\omega_{\max }=v / R$, and expressing the deflection in terms of the axial stress $\sigma=4 H / \pi d^{2}$ in the wire, gives the peak deflection as

$$
\delta_{\mathrm{p}}=\frac{\rho v^{2} L^{2}}{8 R \sigma}
$$

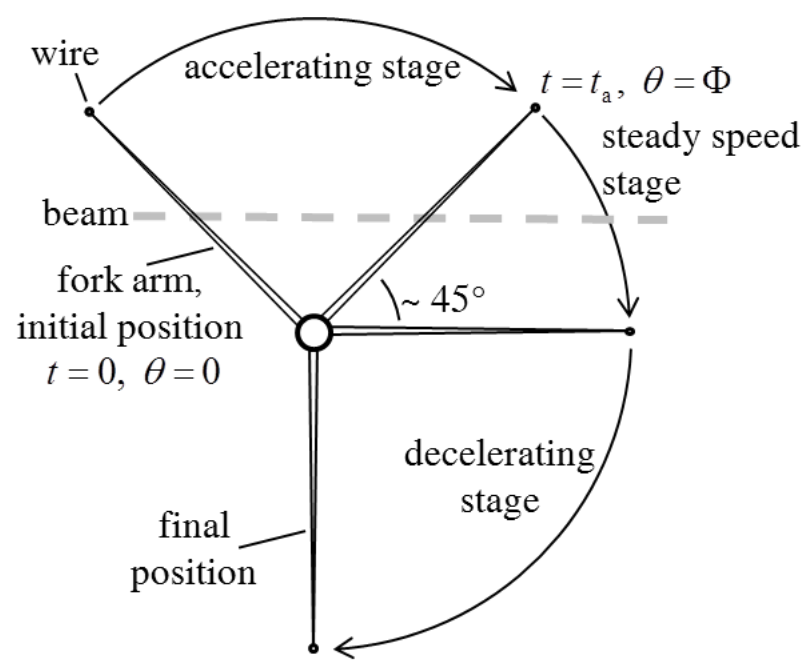

Figure 3. Scanning process: the wire, seen in end view, is brought up to scanning speed in time $t_{\mathrm{a}}$ over an angular range $\Phi$, followed by a steady speed stage and deceleration to rest. 
(a)

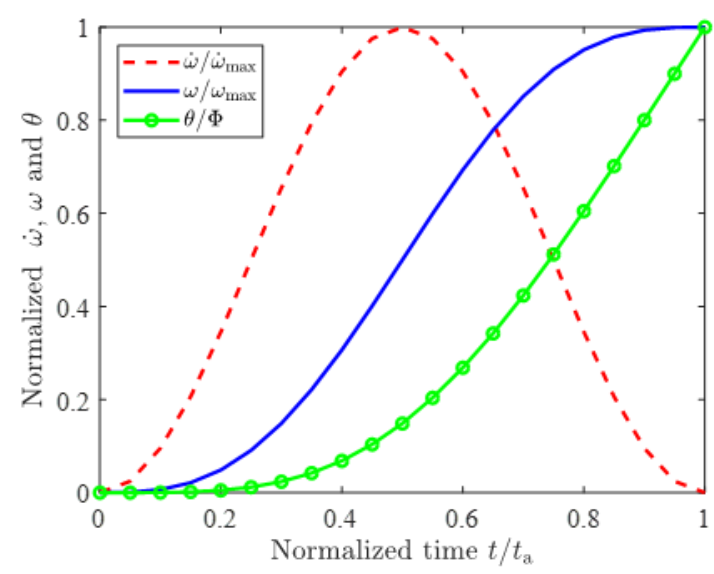

(b)

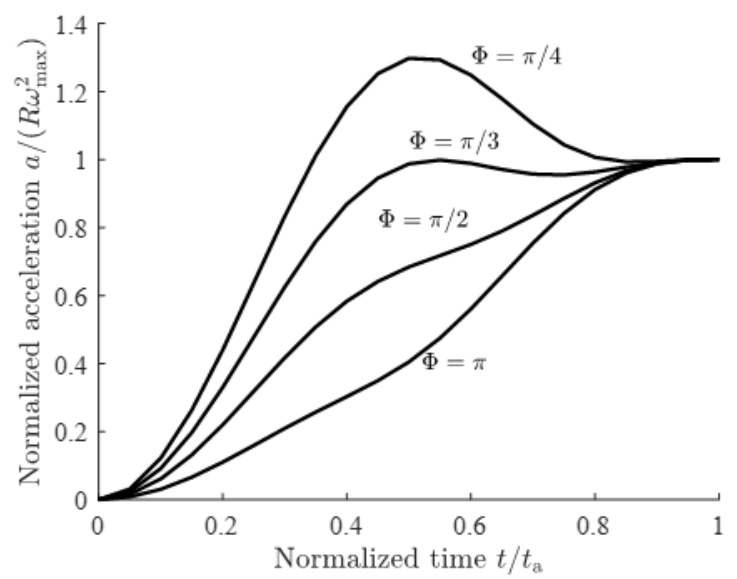

Figure 4. Kinematics of the wire motion as it is brought up to full speed. (a) Angular acceleration $\dot{\omega}$, velocity $\omega$, and position $\theta$, derived from equation (12). (b) Variation of normalized acceleration magnitude with time. This includes both centripetal and tangential terms, and depends on the angular range, $\Phi$, over which the wire is brought up to full speed. For $\Phi>\pi / 3$ the peak acceleration occurs at the end of the range.

\subsection{Material index}

An index for the choice of wire material that allows scanning with maximum beam power can be derived by combining the constraints, thermal model and mechanical model. From equation (14), and the constraints $(3,4)$

$$
\frac{\rho v^{2} L^{2}}{8 R \sigma_{\mathrm{f}}}<\delta_{\mathrm{p}}<\delta_{\max }
$$

This limits the maximum scanning velocity that can be achieved, avoiding excessive deflection or over-tensioning of the wire. Note that increasing the pre-tension of the wire always decreases the peak deflection, and, as discussed above, the inertial loading has negligible effect on the wire tension. Thus it is desirable to pre-tension the wire close to its failure stress, and operate the scanner at a speed close to the deflection limit. Meanwhile, from equation (10) and constraint (2),

$$
T_{\mathrm{p}}=\left(\frac{4 P_{\text {beam }} \Omega}{\pi E D}\right)\left(\frac{1}{v \mathrm{c}_{\mathrm{p}}}\right)<T_{\text {max }}
$$

Treating the scanning velocity $v$ as a free design variable, using (15) and (16) and rearranging for the limit on maximum beam power gives 


$$
P_{\text {beam }}<\left(\frac{\pi E D}{\Omega \sqrt{2}}\right)\left(\frac{\sqrt{R \delta_{\max }}}{L}\right)\left(\mathrm{c}_{\mathrm{p}} T_{\max } \sqrt{\frac{\sigma_{\mathrm{f}}}{\rho}}\right)
$$

Here, the right hand side has been grouped into three brackets indicating, respectively, numerical constants and parameters that cannot be varied, wire scanner design parameters that are not affected by the choice of material, and material properties that could be changed by selecting different materials. Thus a material index is

$$
M=\mathrm{c}_{\mathrm{p}} T_{\max }\left(\frac{\sigma_{\mathrm{f}}}{\rho}\right)^{\frac{1}{2}}
$$

The material with the greatest value of $M$ allows the scanning of hadron beams with the greatest power while avoiding excessive temperature increase, breaking in tension or deflecting too much from a straight configuration.

\section{Discussion}

Equation (18) indicates the relative importance of distinct material properties. A high value of specific heat capacity is advantageous in limiting the temperature rise. Combining this with a high value of the maximum tolerable temperature increases the ability of the material to tolerate thermal energy input without failure. The value of $\mathrm{c}_{\mathrm{p}} T_{\max }$ thus represents a material-dependent energy per unit mass that should be maximized provided this does not compromise strength or density. High values of failure stress allow the material to be tensioned more, keeping its deflection within acceptable limits while accommodating greater scanning speeds, which in turn reduces exposure to the beam. But the resulting performance in terms of tolerable beam power scales only as $\sigma_{\mathrm{f}}^{1 / 2}$ because of the quadratic relationship between scanning speed and tensile stress in the wire at the same peak deflection, given by equation (14). Similarly, the material index decreases with increasing material density, scaling as $\rho^{-1 / 2}$. This scaling arises from a combination of factors: greater mass density reduces the temperature rise per unit absorbed thermal energy, but at the same time, increases the rate of energy absorption from the beam. This happens because the beam effectively immerses a portion of the wire in high energy hadrons that deposit energy approximately in proportion to the quantity of matter encountered. The mass density also affects the inertial loading due to the acceleration of the wire. The balance between these effects gives the inverse square root dependency on mass density, such that materials with a wide range of density may make good candidates. In existing scanner 
designs, both carbon wires, density $2200 \mathrm{~kg} \mathrm{~m}^{-3}$, and tungsten wires, density $19300 \mathrm{~kg} \mathrm{~m}^{-3}$, have been used. ${ }^{[11]}$ Material indices of the form $\sigma_{f}^{k} / \rho$ are common in minimum weight or inertial loading structural design problems, with $k=1$ for the design of lightweight tie rods and flywheels, $k=2 / 3$ for minimum weight strong beams and shafts, and $k=1 / 2$ for minimum weight strong panels. Here, the strength-density combination is equivalent to $k=1$, which favours high strength and low density materials, making engineering ceramics likely candidates. The brittle nature of these materials commonly prohibits applications in tensile loading, but here, the fine fibre geometry is advantageous in limiting the size of incipient flaws, $l_{f}$ (note: strength scales with $l_{f}^{-1 / 2}$ ) and therefore in enhancing strength.

Materials Data for $\mathrm{c}_{\mathrm{p}}, T_{\max }, \sigma_{\mathrm{f}}$ and $\rho$ were gathered from a wide variety of sources. ${ }^{[9,12-15]}$ These data are presented in figure 5, along with a guide line for selection of high performance materials for the wire scanner. All materials that lie along the guide line indicated have the same value of the merit index, given by equation (18), as carbon. Any material that lies above this line has a higher merit index and is potentially a better material than carbon for this application. Some care is needed in defining the suitable property values for each material. For many materials or classes of materials, this is dealt with by attributing a range of values to each material property, based on the upper and lower limits found in the literature. The specific heat capacity, $c_{p}$, varies significantly with temperature for many materials. This was accounted for by including a range based on the value at $300 \mathrm{~K}$ as a lower limit, and the value at the Debye temperature, where known, as an upper limit. In materials with a Debye temperature less than about $400 \mathrm{~K}$ there is little change in heat capacity during the heating process. However, some extremal materials such as carbon have Debye temperatures in excess of $2000 \mathrm{~K}$, in which case the heat capacity can increase tenfold with increasing temperature. For the purpose of comparing materials in figure 5 , the $T_{\max }$ value was set equal to the melting point $T_{\mathrm{m}}$ of each material, with a few exceptions. High temperature softening means that a maximum service temperature in the range $0.6-0.9 T_{\mathrm{m}}$ is realistic, with the lower end of this range most relevant to ductile metals, and the upper end for refractory ceramics. Once again, a range of recorded melting points was noted in the literature, for several materials and material classes. Carbon is exceptional in having no melting point at low pressure, due to sublimation; $T_{\max }$ was set to the sublimation temperature in this case. 


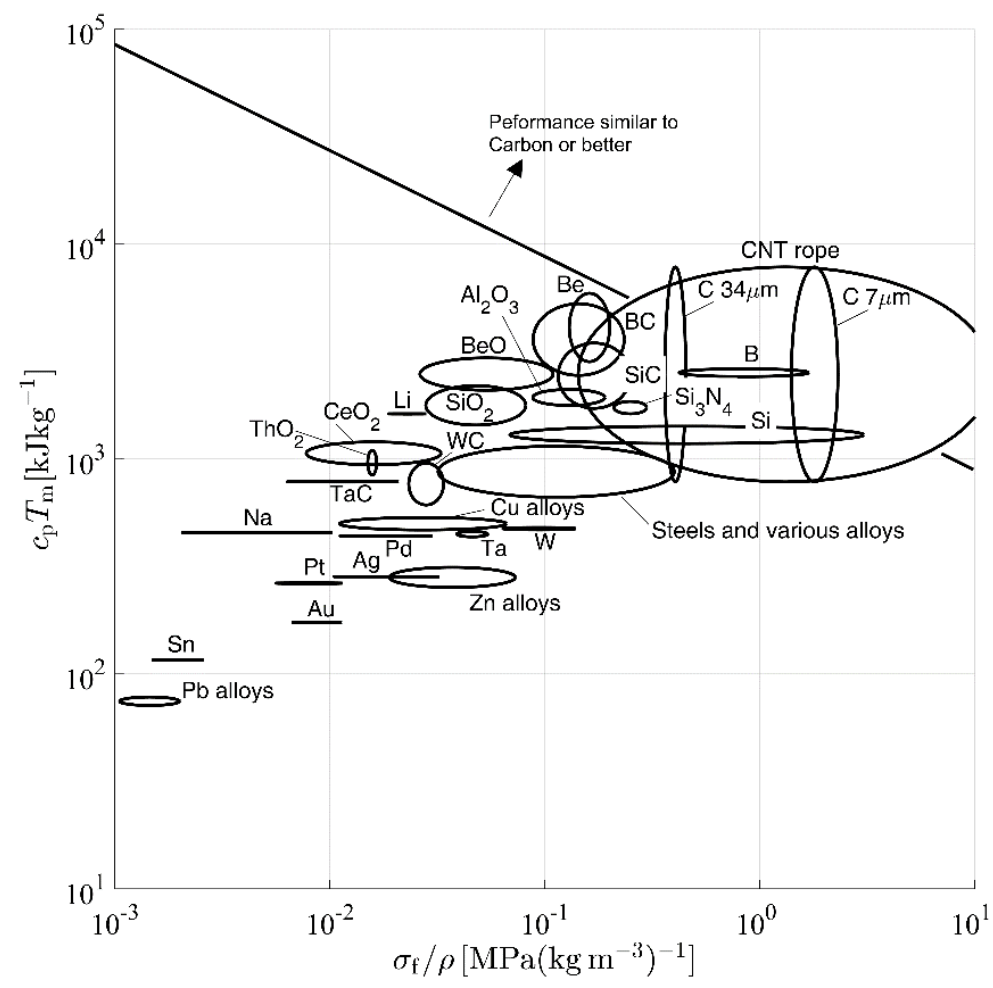

Figure 5. Materials selection chart for wires in the LHC. Many engineering alloys cluster around the performance level of steels and have been omitted for clarity. Labels refer to: "CNT rope" - carbon nanotube rope, "C $34 \mu \mathrm{m}$ " - $34 \mu \mathrm{m}$ carbon fibre, "C $7 \mu \mathrm{m}$ " - $7 \mu \mathrm{m}$ carbon fibre. Other labels give the chemical elements or compounds.

Figure 5 gives a very clear indication of the suitability of carbon for the wires: carbon fibres have a remarkable combination of heat capacity, strength, high maximum service temperature and low density that is matched by very few materials. Carbon also offers a wide range of performance since different fibre diameters, with somewhat different properties, are readily available. The wide range of $c_{\mathrm{p}} T_{\mathrm{m}}$ values for carbon results from uncertainty over the most representative value of $c_{p}$, given the great change in heat capacity with temperature. Performance close to the centre of this range $\left(\mathrm{c}_{\mathrm{p}} T_{\mathrm{m}} \sim 3 \times 10^{3} \mathrm{~kJ} \mathrm{~kg}^{-1}\right)$ is likely. Competitor materials include boron, beryllium (undesirable because of toxicity) and boron carbide, all of which are available in the form of fibres. Carbon nanotube ropes, consisting of bundles of nanotubes spun into fibres, provide another alternative. Here, the materials provide a very wide potential range of strength performance based on claims in the literature. At the top end of the strength range, this could give advantageous performance, provided the corresponding increase in scanning speeds can be realized. Several engineering ceramics (alumina, silicon nitride, 
silicon carbide) and pure silicon, would be worth exploring, provided they can be obtained in fibre form. Prior attempts at using ceramic fibres have encountered difficulty due to charging of the wire; electrical conductors are preferable in this respect. For example, SiC was tested as a wire material, ${ }^{[16,17]}$ but failed at temperatures below those expected, so some other failure mechanisms may have become dominant, such as sublimation or surface charging effects.

The analysis also indicates some directions that are likely to be worth exploring for enhanced performance, and some that are not. Approaches that enhance the heat capacity over a range of temperature could lead to direct performance improvements. This motivates a search for materials with solid-solid phase transformations having a high value of latent heat. Some iron cobalt and iron nickel alloys ${ }^{[18]}$ exhibit phase transitions with enthalpy of around $50 \mathrm{~kJ} \mathrm{~kg}^{-1}$; however, this is insufficient to make them competitive for the wire scanner application. Greater values of solid-state enthalpy change can be achieved in organic materials, but these would be unsuitable due to volatility under vacuum. Other considerations could include pre-cooling of the wire or the use of fine whiskers to approach the ideal strength. Other forms of carbon, such as graphene nanoribbons, have attracted great attention but it remains unclear whether these materials could be made into a practical wire, and what would be the resulting material properties.

Several simplifications were made in the analysis. A feature of the Ashby material index is that it is insensitive to details of the analysis, such as the exact beam shape or particle distribution. Using a more realistic beam distribution, such as a truncated Gaussian, would alter the peak temperature reached at a given scan velocity, and so could affect the maximum beam power that can be tolerated. However, this effect would apply similarly to all materials, and so the material index for selection of the most suitable wire material would be unchanged. Thus the analysis, though simplified, is relevant to real applications.

The analysis suggests that modifications to the emissivity, thermal conductivity or mass density of the wire are unlikely to have significant impact on performance. Hence high emissivity coatings, composites with high conductivity components or structural modifications, such as porosity, are likely to yield only limited gains. 


\section{Conclusion}

The problem of selecting a wire material for the wire scanners in the Large Hadron Collider has been analyzed from the perspective of Ashby material indices. An unusual problem from the point of view of engineering materials selection, the analysis involves mechanical, thermal and nuclear interaction properties. Nevertheless, with suitable approximations, a simple material index depending only on melting temperature, heat capacity, strength and density was derived. This allows the comparison of performance over a wide range of materials. Perhaps unsurprisingly, carbon, which is currently used for this application, was found to offer extremely good performance. Some ceramic materials worthy of further investigation were identified, and the analysis provides guidance for routes towards improved performance. Analysis of this kind is currently valuable to the beam instrumentation community as higher energy and intensity accelerators are being designed. Wire scanners have proven invaluable for direct measurement of the transverse beam profile. Even if the limits of available materials make them no longer able to scan the full intensity of the LHC beam, they are still the principal instrument for calibration of less invasive, but less direct instruments based on synchrotron light or beam-gas interactions. As such, identification of new materials with the potential to improve the intensity reach of wire scanners will ensure their use for the next generation of particle accelerators. More generally, analysis of this kind enables a preliminary selection of candidate materials with potential in applications where performance is critical and the physics involved can make some conventional material choices uncompetitive.

\section{Acknowledgements}

This work resulted from a longstanding collaboration between the University of Oxford and CERN which has been supported by CERN and members of the CERN beam instrumentation group. The authors are grateful to Anton Lechner for carrying out FLUKA analyses, and also recognize contributions from project students Michael Zhang and Man Yuan. The authors are also grateful for comments on the manuscript from Rhodri Jones.

\section{References}

[1] G. Apollinari, O. Bruening, T. Nakamoto, L. Rossi, in High-Luminosity Large Hadron Collider (HL-LHC), in CERN Yellow Report CERN-2015-005, 2015, 1-19.

[2] S. Eidelman et al., Passage of particles through matter Physics Letters B592, 2004, 1.

[3] J. Emery, B. Dehning, C. Morais Pereira, J. Sirvent Blasco, S. Cantin, M. Tognolini, B. Schneider, K. Henzer, M. Starkier. A fast and accurate wire scanner instrument for the CERN accelerators to cope 
with severe environmental constraints and an increased demand for availability, in Proceedings of 2014 IEEE Conference on Control Applications (CCA), October 8-10, 2014. Antibes, France

[4] M.F. Ashby. On the engineering properties of materials. Acta Metall. 1989 37, 1273-1293.

[5] M.F. Ashby. Materials selection in mechanical design. 3rd edition, Elsevier ButterworthHeinemann, 2005.

[6] S. Myers, E. Picasso, E. The design, construction and commissioning of the CERN large ElectronPositron collider. Contemp. Phys. 1990, 31, 387-403.

[7] M. Sapinski. Model of Carbon Wire Heating in Accelerator Beam. CERN internal report CERNAB-2008-030, 2008.

[8] M.F. Ashby. Materials and the Environment. Eco-informed material choice. Elsevier ButterworthHeinemann,, 2009.

[9] C. Patrignani (Particle Data Group), Review of Particle Physics, Chinese Physics C, 2016, 40, 100001. http://pdg.lbl.gov/index.html

[10] G. Battistoni, T. Boehlen, F. Cerutti, P. Wai Chin, L. Esposito, A. Fassò, A. Ferrari, A. Lechner, A. Empl, A. Mairani, A. Mereghetti, P. Garcia Ortega, J. Ranf, S. Roesler, P.R. Sala, V. Vlachoudis, G. Smirnov. Overview of the FLUKA code. Annals of Nuclear Energy 2015, 82, 10-18.

[11] B. Cheymol, A. Jansson, T. Shea. Wire scanner design for the European spallation source, in Proceedings of IBIC2013, Oxford, UK. 2013

[12] C. Kittel. Introduction to Solid State Physics, 7th Ed., Wiley. 1996

[13] P.J. Linstrom, W.G. Mallard, Eds. NIST Chemistry WebBook, NIST Standard Reference Database Number 69, National Institute of Standards and Technology, Gaithersburg MD, 20899, doi:10.18434/T4D303

[14] Engineer's handbook. The Engineer's Handbook. http://engineershandbook.com/

[15] O.D. Neikov, S.S. Naboychenko, N.A. Yefimovet. Handbook of Non-Ferrous Metal Powders: Technologies and Applications. Elsevier, Amsterdam, 2009.

[16] F. Caspers, B. Dehning, E. Jensen, J. Koopman, J.F. Malo, F. Roncarolo. Cavity mode related wire breaking of the SPS wire scanners and loss measurements of wire materials, in Proceedings of DIPAC 2003, Mainz, Germany. 2003

[17] J.F. O'Hara, J.D. Gilpatrick, L.A. Day, J.H. Kamperschroer, D.W. Madsen. Slow wire scanner beam profile measurement for LEDA. AIP Conference Proceedings 2000, 546, 510-518.

[18] A. Fallahi, G. Guldentops, M. Tao, S. Granados-Focil, S. Van Dessel. Review on solid-solid phase change materials for thermal energy storage: Molecular structure and thermal properties. Appl. Therm. Eng., 2017, 127, 1427-1441. 